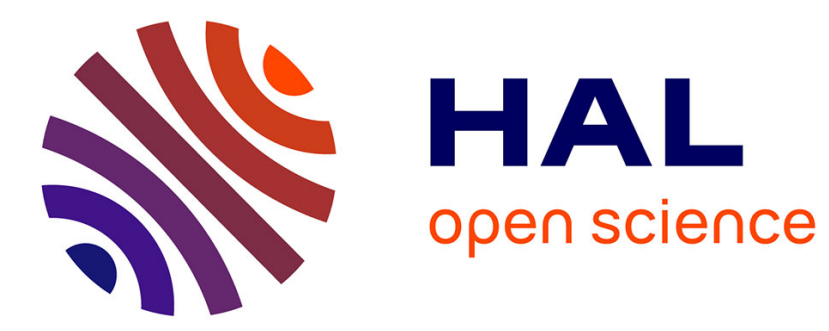

\title{
Une méthode simple de prévision des températures de l'air et de la surface du sol en conditions de gelées radiatives
}

\author{
Pierre Cellier
}

\section{- To cite this version:}

Pierre Cellier. Une méthode simple de prévision des températures de l'air et de la surface du sol en conditions de gelées radiatives. Agronomie, 1984, 4 (8), pp.741-747. hal-00884692

\section{HAL Id: hal-00884692 \\ https://hal.science/hal-00884692}

Submitted on 1 Jan 1984

HAL is a multi-disciplinary open access archive for the deposit and dissemination of scientific research documents, whether they are published or not. The documents may come from teaching and research institutions in France or abroad, or from public or private research centers.
L'archive ouverte pluridisciplinaire HAL, est destinée au dépôt et à la diffusion de documents scientifiques de niveau recherche, publiés ou non, émanant des établissements d'enseignement et de recherche français ou étrangers, des laboratoires publics ou privés. 


\section{Une méthode simple de prévision des températures de l'air et de la surface du sol en conditions de gelées radiatives}

Pierre CELLIER

I.N.R.A., Laboratoire de Bioclimatologie, Route de St-Cyr, F 78000 Versailles

\begin{abstract}
A partir d'un modèle unidimensionnel de calcul de l'évolution nocturne de la température de l'air et du sol, on a tracé des abaques qui expriment le refroidissement en conditions de ciel clair, en fonction de différentes données d'entrée, relevées au coucher du soleil. Celles-ci sont, à l'exception du rayonnement net, des mesures effectuées couramment au parc météorologique : vitesse du vent à $2 \mathrm{~m}$, températures de l'air, du point de rosée et du sol à $10 \mathrm{~cm}$. Les abaques sont décrits par des équations simples qui permettent d'établir une prévision une heure après le coucher du soleil.

Les résultats s'avèrent être aussi bons que ceux qui sont obtenus pour les mêmes cas avec d'autres méthodes classiques (YOUNG, LUTERSTEIN-CHUDNOVSK1). La méthode présente les avantages de n'utiliser aucun paramètre local et de donner une qualité de prévision semblable pour la température de l'air et celle de la surface du sol. Elle pourrait avantageusement être appliquée en combinaison avec d'autres méthodes basées sur des principes très différents.
\end{abstract}

Mots clés additionnels : Gelées de printemps, parc météorologique, modèle numérique, rayonnement net, vent.

\begin{abstract}
A simple method for air and soil surface temperature prediction under radiative frost conditions.
A one-dimensional model of nocturnal change in soil and air temperature has been used to plot a curve describing cooling under clear sky conditions in relation to measurements made at sunset. Apart from net radiation measurement, these are classical meteorological data : wind speed at $2 \mathrm{~m}$, air and soil temperature, dew point. The curves could be described by simple equations, used to make a prediction one hour after sunset. The results were as good as those obtained with other methods (YounG, LUTERSTEIN-CHUDNOvSKi). No local parameter was introduced and the quality of prediction was similar for soil surface and air temperature. The method could be applied combined with others, based upon different principles.
\end{abstract}

Additional key words : Spring frost, meteorological ground, numerical model, net radiation, wind.

\section{INTRODUCTION}

C'est au voisinage de la floraison que les risques de dégâts dus aux gelées sur les arbres fruitiers sont les plus graves (DURAND, 1978). Les arboriculteurs sont alors demandeurs de prévisions des températures minimales nocturnes, qui leur donneront les critères nécessaires à la décision de mise en œuvre de leurs moyens de lutte contre les gelées (aspersion, machines à vent, chaufferettes, brouillards artificiels, ...) (TABARD, 1978 ; de VILLELE, 1980). Ces prévisions doivent être disponibles le plus tôt possible (au plus tard 19-20 h) afin de leur permettre de faire face à l'éventuelle gelée (mobilisation du personnel, préparation du matériel).
De son côté, le prévisionniste dispose actuellement de diverses méthodes d'estimation de la température minimale nocturne :

- formules hygrométriques (ELLISON, 1928 ; SUTTON, 1953 ; GEORG, 1978) qui n'utilisent, en général, qu'une mesure de température et d'humidité de l'air sous abri, au coucher du soleil.

- formule de BRUNT, ou des adaptations (BRUNT, 1941 ; JAEGER, 1945 ; GROEN, 1947 ; SUTTON, 1953 ; Gerbier \& PEDEAU, 1974) qui utilisent, outre des mesures de température et humidité de l'air sous abri, des corrections destinées à prendre en compte les conditions de sol et la vitesse du vent prévisible en cours de nuit. L'adaptation qui a été faite par la Météorologie 
Nationale (GERBIER \& PEDEAU, 1974) permet une estimation dès $15 \mathrm{~h}$ TU de l'indice actinothermique minimal, qui donne une température proche de celle des bourgeons.

- méthode de SAUNDERS (1952) adaptée par BARTHRAM (1964), à partir de laquelle la Météorologie Nationale a élaboré à 2 méthodes de prévision de la température minimale, l'une dite "synoptique " estimation de la température minimale d'une masse d'air homogène - l'autre dite "locale » qui utilise une mesure prise sous abri, ainsi qu'un terme advectif. Elles sont toutefois de mise en œuvre assez complexe, demandant une connaissance du type et de la structure de la masse d'air. En outre, elles ignorent totalement les processus de surface, qui sont pourtant prédominants dans le cas des gelées radiatives.

Depuis les années 70 , du fait d'améliorations très importantes dans la connaissance des échanges d'énergie au voisinage de la surface du sol, principalement par convection, et poussés par le progrès de l'informatique, quelques modèles décrivant de façon beaucoup plus précise le processus de refroidissement ont vu le jour (GeORG, 1978; Sutherland, 1980 ; Cellier \& ITIER, 1984). Ce sont, toutefois, des modèles complexes peu accessibles au prévisionniste sous leur forme complète, qui utilisent quelquefois des mesures non disponibles de façon courante (température de l'air à $1,5 \mathrm{~m}$, $3 \mathrm{~m}$ et $9 \mathrm{~m}$, pour le modèle de SUTHERLAND, par exemple).

La méthode simple de prévision de la température de la surface du sol et dans l'air sur les premiers mètres, à partir de mesures réalisées au parc météorologique, que nous allons présenter ici est une sortie simplifiée d'un tel type de modèle (CELlier, 1982 ; CELliER \& ITIER, 1984).

\section{LE MODÈLE DE REFROIDISSEMENT NOCTURNE}

\section{A. Présentation sommaire}

Le modèle calcule le refroidissement dans un système compris entre une profondeur de $1 \mathrm{~m}$ dans le sol et une altitude de $200 \mathrm{~m}$ dans l'atmosphère, divisé en couches horizontales homogènes d'autant plus fines qu'elles sont plus proches de la surface (fig. 1). Il utilise un schéma unidimensionnel de calcul numérique en différences finies, de type explicite. A partir de 5 données d'entrée relevées au voisinage de la surface du sol (voir II.B), il établit un profil initial dans l'ensemble du système (température et hygrométrie de l'air, température du sol) et calcule certains paramètres extérieurs au système (vent géostrophique, température radiative apparente du ciel à $1000 \mathrm{~m}$ ), caractéristiques de la masse d'air, qui sont gardés constants tout au long de la nuit eu égard à la stabilité des conditions météorologiques habituellement rencontrées (situation anticyclonique). L'évolution de la température et d'autres paramètres d'échange est ensuite entièrement générée par le modèle lui-même, qui la calcule par application du principe de conservation de l'énergie à chaque couche :

- à la surface du sol, ce principe est traduit par l'équation du bilan d'énergie ; son importance est pri-

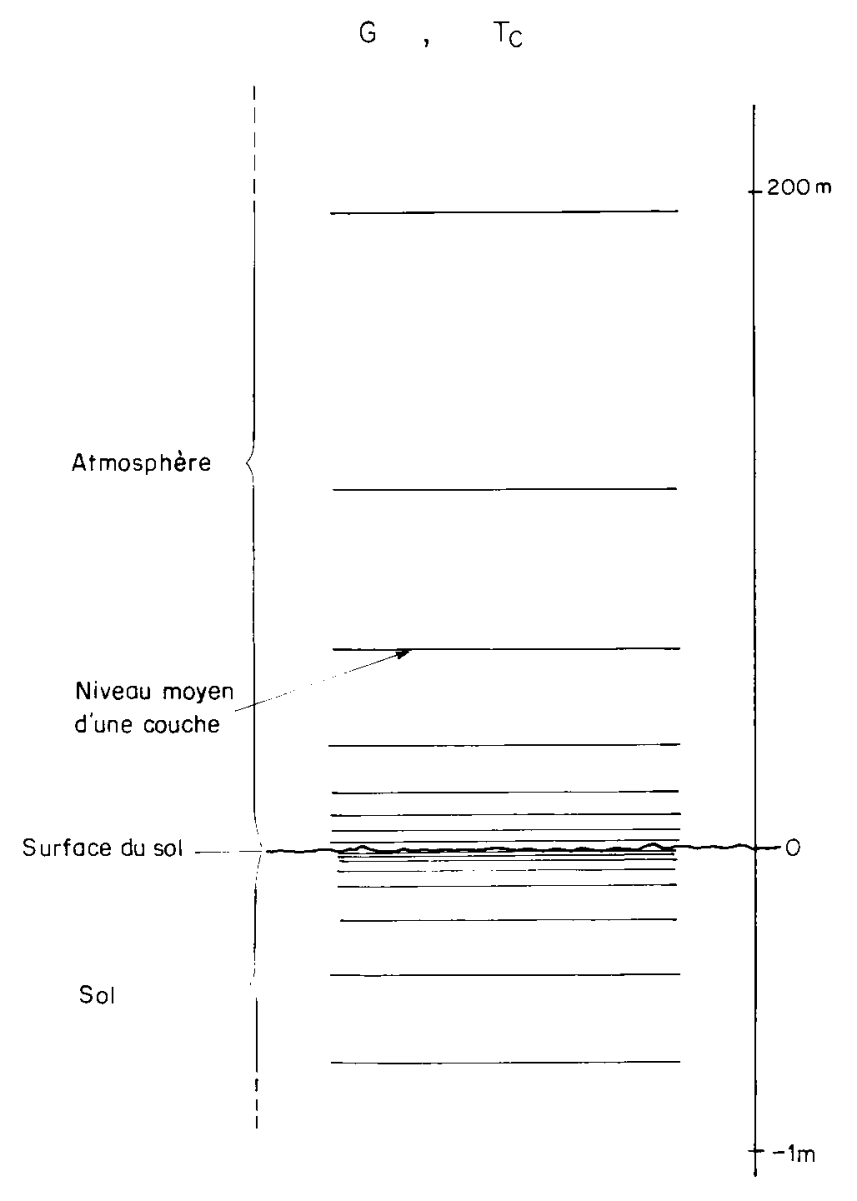

Figure 1

Schéma du système utilisé dans le modèle numérique complet. $G=$ vent géostrophique;

$T c=$ température radiative apparente du ciel à $1000 \mathrm{~m}$.

Schematization of system used by the complete numerical model. $G=$ geostrophic wind

$T c=$ apparent radiative temperature of sky at $1000 \mathrm{~m}$.

mordiale puisque c'est le déficit radiatif qui initie le refroidissement dans l'ensemble du système,

- dans les couches de sol, le refroidissement est transmis vers la profondeur par conduction,

- dans les couches atmosphériques, les transferts convectifs et radiatifs sont intégrés sous forme de schémas simplifiés.

Les apports d'énergie par condensation/congélation de la vapeur d'eau sont également pris en compte, ainsi que la modification du bilan radiatif causée par l'apparition de brouillard naturel.

\section{B. Les données d'entrée du modèle}

\section{Rayonnement net, $R n$}

La cause principale des gelées de printemps est l'existence d'un fort déficit radiatif au niveau de la surface $\mathrm{du}$ sol et des organes végétaux. L'importance de ce terme justifie qu'on utilise comme donnée prédictive une mesure directe du rayonnement net. Toutefois celle-ci n'est pas couramment effectuée au parc météorologique et, le cas échéant, il sera possible d'estimer le rayonnement net à partir de mesures de la température et de l'humidité de l'air en utilisant les formules exis- 
tantes. En particulier, à l'échelle horaire, dans les conditions particulières de début de nuit, la formule de BRUTSAERT (1975), supposée universelle, donne de bons résultats pour l'estimation du rayonnement atmosphérique, Ra, en conditions de ciel clair. Rs, étant le rayonnement émis par le sol, on estime le rayonnement net $\mathrm{Rn}=\mathrm{Ra}-\mathrm{Rs}$ par :

$$
\mathrm{Rn}=\mathcal{E}_{\mathrm{a}}^{\prime} \sigma \mathrm{T}_{\mathrm{a}}^{\prime 4}-\varepsilon_{\mathrm{s}} \sigma \mathrm{T}_{\mathrm{s}}^{4}
$$

$\varepsilon_{\mathrm{a}}^{\prime}=1,24\left(\frac{\mathrm{ea}}{T_{\mathrm{a}}^{\prime}}\right)^{1 / 7}$

= émissivité apparente de l'atmosphère (formule de BRUTSAERT)

ea $=$ pression de vapeur $(\mathrm{mb})$ sous abri à $2 \mathrm{~m}$.

$\mathrm{T}_{\mathrm{a}}^{\prime}=$ température absolue de l'air $(\mathrm{K})$ sous abri à $2 \mathrm{~m}$.

$\varepsilon_{\mathrm{s}}=$ émissivité de la surface du sol (on prend une valeur moyenne $\varepsilon_{\mathrm{s}}=0,98$ ).

$\mathrm{T}_{\mathrm{s}}=$ température de la surface du sol $(\mathrm{K})$; on choisit $\mathrm{T}_{\mathrm{s}}=\mathrm{T}_{\mathrm{a}}^{\prime}$ au-dessus d'un sol nu ;

$T_{s}=T_{a}^{\prime}-2$ si le sol est recouvert d'une végétation couvrant plus de la moitié de la surface du sol.

$\sigma=$ constante de Stephane - Boltzmann $\left(5,67 \cdot 10^{-8} \cdot \mathrm{W} \cdot \mathrm{m}^{-2} \cdot \mathrm{K}^{-4}\right)$.

Sur 48 points de comparaison entre mesures et estimations de Rn à l'aide des formules (1) et (2), l'écarttype des écarts à la droite de régression est égal à $6 \mathrm{Wm}^{-2}$.

Dans tous les cas, les mesures sont effectuées $1 \mathrm{~h}$ après le coucher du soleil, moment où le rayonnement net (négatif) est proche de son minimum.

\section{Caractérisation de la masse d'air}

\section{a) Température et humidité de l'air}

La même mesure d'humidité de l'air que dans la partie précédente est utilisée, exprimée cette fois-ci sous la forme de température du point de rosée, $\mathrm{Td}(\mathrm{ea}=$ pression de vapeur saturante à la température Td). Elle permet d'estimer la limitation du refroidissement, par les processus de condensation/congélation, lorsque la température tombe sous celle du point de rosée.

La température de l'air sous abri au coucher du soleil, Ta, est prise comme température initiale de l'air et de la surface du sol.

\section{b) Vitesse du vent}

On observe aisément que le refroidissement nocturne est moins intense lorsque le vent est fort, au cours de la nuit. Cette limitation est caractérisée par une mesure de la vitesse du vent à $2 \mathrm{~m}$, au coucher du soleil, $\mathrm{V}\left(\mathrm{ms}^{-1}\right)$. Il sera préférable d'utiliser une moyenne sur une $1 / 2 \mathrm{~h}$ ou $1 \mathrm{~h}$ avant le coucher de soleil, afin de s'affranchir, au moins partiellement, de la forte variabilité temporelle de cette mesure.

\section{Caractérisation du sol}

La quantité d'énergie restituée par le sol par conduction est caractérisée par l'écart entre la température de l'air, $\mathrm{Ta}$, et la température du sol à $10 \mathrm{~cm}$ (notée $\mathrm{T}_{\mathrm{S} 10}$ ), mesurées au coucher du soleil. En effet, la température du sol à $10 \mathrm{~cm}$, relevée au coucher du soleil, est d'autant plus élevée par rapport à la température de l'air que la quantité d'énergie stockée dans le sol pendant la journée précédente est importante, et que la capacité calorifique volumique du sol est plus faible.

L'ensemble des données d'entrée, ainsi que leur heure de relevé sont récapitulés sur la figure 2 .

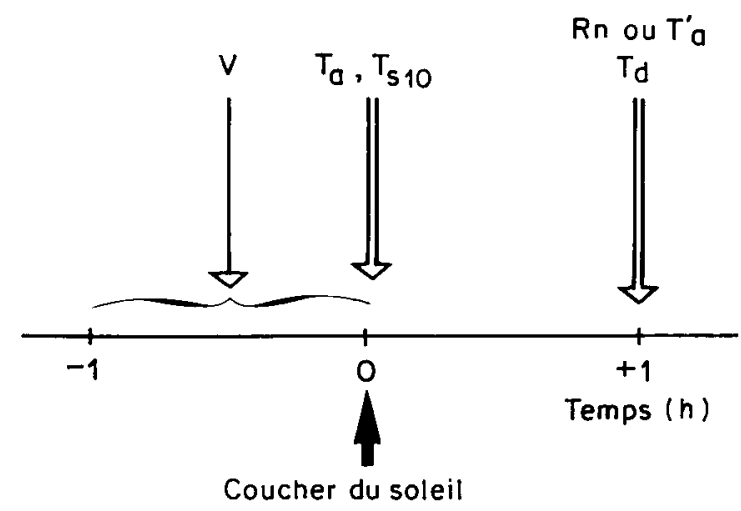

Figure 2

Mesures utilisées comme données d'entrée de la méthode simplifiée et leur heure de relevé.

Time and type of measurement of input data used in the simple method.

\section{LA MÉTHODE SIMPLIFIÉE}

\section{A. Elaboration et principe de la méthode simplifiée}

La méthode simplifiée que nous proposons dérive du modèle numérique ci-dessus et utilise les mêmes variables d'entrée ( $\mathrm{Rn}, \mathrm{Ta}, \mathrm{Td}, \mathrm{T}_{\mathrm{S} 10}$, et $\left.\mathrm{V}\right)$. En imposant à celles-ci différentes combinaisons plausibles de valeurs et en étudiant, par simulation à l'aide du modèle numérique, le refroidissement nocturne, on a cherché à exprimer la température minimale atteinte comme fonction des variables d'entrée :

$$
\operatorname{Tmin}(\mathrm{z})-\mathrm{Ta}=\mathrm{f}\left(\mathrm{Rn}, \mathrm{Ta}, \mathrm{Td}, \mathrm{T}_{\mathrm{S} 10}, \mathrm{~V}\right)
$$

pour les hauteurs $\mathrm{z}=0$ (surface du sol), $\mathrm{z}=1,5 \mathrm{~m}$ et $\mathrm{z}=3,0 \mathrm{~m}$.

Cette fonction a finalement été explicitée sous la forme :

$$
\begin{aligned}
& \mathrm{Tmin}(\mathrm{z})-\mathrm{Ta}=(\mathrm{A} 1+\mathrm{A} 2+\mathrm{A} 3) \ln (\mathrm{dn}) \\
& \mathrm{dn}: \text { durée de la nuit }(\mathrm{en} \mathrm{h}) ;
\end{aligned}
$$

A1, A2, A3 : coefficients ayant la dimension d'une température $\left({ }^{\circ} \mathrm{C}\right)$.

- A1 : traduit l'influence des transferts radiatifs/ convectifs sur le refroidissement. Il dépend de $\mathrm{Rn}, \mathrm{V}$ et de la hauteur $\mathrm{z}$ considérée (fig. 3 ) et peut sans difficulté être calculé par :

$$
\mathrm{A} 1=\mathrm{a}+\mathrm{b} . \mathrm{V}
$$

$a$ et $b$ : coefficients dont l'expression est donnée dans le tableau 1.

- A2 : précise la partition du déficit radiatif entre le sol et l'atmosphère ; fonction de $\mathrm{Ta}, \mathrm{T}_{\mathrm{S} 10}$, $\mathrm{V}$, il est pra- 


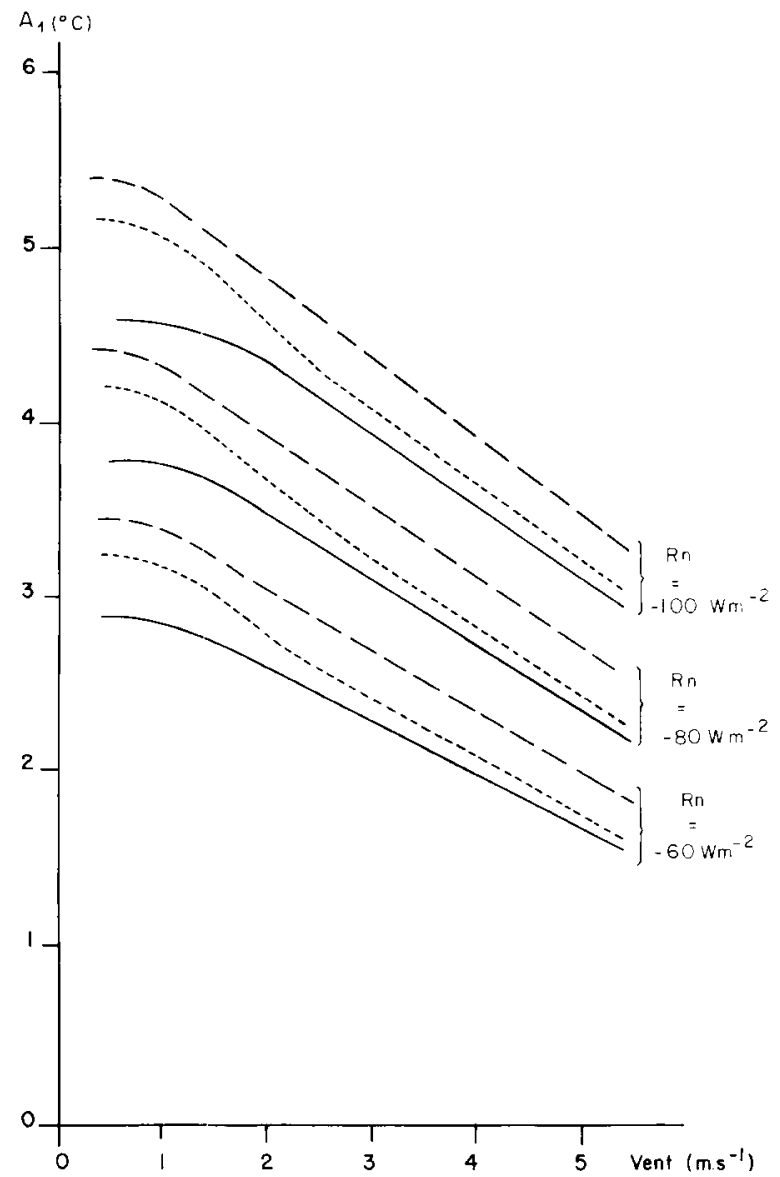

Figure 3

Abaques décrivant la variation du facteur $A I$ de la relation (4) en fonction des données d'entrée $R n$ et $\mathrm{V}$.

(__ $3 \mathrm{~m}$, -.-. $1.5 \mathrm{~m}$; - - surface du sol).

Variation of the Al factor in relation (4), relative to input data $R n$ and $V$.

(__ $3 \mathrm{~m}, \ldots . .-1.5 \mathrm{~m}$; _ soil surface).

tiquement indépendant de la hauteur z (fig. 4). On peut le calculer par :

$$
\mathrm{A} 2=0,024\left(\mathrm{Ta}-\mathrm{T}_{\mathrm{S} 10}\right)(10-\mathrm{V})
$$

- A3 : terme correctif dépendant de Ta $\left({ }^{\circ} \mathrm{C}\right)$ :

$$
\mathrm{A} 3=0,04(\mathrm{Ta}-12)
$$

Si la température $\operatorname{Tmin}(\mathrm{z})$ calculée à l'aide des équations (1) à (4) est inférieure à $T d$, une correction, destinée à prendre en compte la production de chaleur par condensation, s'ajoute et conduit à :

$$
\mathrm{T}^{\prime} \min (\mathrm{z})=\operatorname{Tmin}(\mathrm{z})-\frac{\mathrm{P}^{\prime}}{\mathrm{P}^{\prime}+\gamma}(\operatorname{Tmin}(\mathrm{z})-\mathrm{Td})
$$

où $T^{\prime} \min$ est la prévision définitive

$\gamma:$ constante psychrométrique $=66\left(\mathrm{~Pa}{ }^{\circ} \mathrm{C}^{-1}\right) ;$

$\mathrm{P}^{\prime}$ : dérivée de la courbe de pression de vapeur saturante, à la température $(\mathrm{Td}+\operatorname{Tmin}(\mathrm{z})) / 2$. $\left(\mathrm{Pa}{ }^{\circ} \mathrm{C}^{-1}\right)$.

On utilisera $\mathrm{P}^{\prime}=45+3(\mathrm{Td}+\mathrm{Tmin}(\mathrm{z})) / 2$

\section{B. Concordance entre la méthode simplifiée et le modèle numérique}

La figure 5 montre que la méthode simplifiée retrace tout à fait correctement les résultats obtenus à l'aide du

\section{TABLEAU 1}

Expression des coefficients a et $b$ de la formule (5) en fonction des données d'entrée $R n\left(\mathrm{Wm}^{-2}\right)$ et $\mathrm{V}\left(\mathrm{ms}^{-1}\right)$ pour la prévision des températures minimales à différentes hauteurs au-dessus du sol.

Expression of the coefficients $a$ and $b$ in formula (5) in relation to the input data $R n\left(\mathrm{Wm}^{-2}\right)$ and $V\left(\mathrm{~ms}^{-1}\right)$ for predicting temperature minimum at different heights above soil level.

\begin{tabular}{cccc}
\hline \multirow{2}{*}{$\begin{array}{c}\text { Hauteur } \\
\text { au-dessus } \\
\text { du sol }\end{array}$} & $\begin{array}{c}\text { Gamme de } \\
\text { vitesse } \\
\text { du vent } \\
\left(\mathrm{ms}^{-1}\right)\end{array}$ & $\begin{array}{c}\text { Expression du } \\
\text { coefficient } \mathrm{a}\end{array}$ & $\begin{array}{c}\text { Expression du } \\
\text { coefficient } \mathrm{b}\end{array}$ \\
\hline $\begin{array}{c}\text { Surface } \\
\text { du sol }\end{array}$ & $\mathrm{V}<1$ & $-0,050 \mathrm{Rn}+0,56$ & 0 \\
\cline { 2 - 4 } & $\mathrm{V}>1$ & $-0,050 \mathrm{Rn}+0,81$ & $0,0025 \mathrm{Rn}-0,24$ \\
\hline & $\mathrm{V}<1$ & $-0,047 \mathrm{Rn}+0,40$ & 0 \\
\hline & $\mathrm{V}>1$ & $-0,050 \mathrm{Rn}+0,60$ & $0,0027 \mathrm{Rn}-0,23$ \\
\hline $3,5 \mathrm{~m}$ & $\mathrm{~V}<1,5$ & $-0,043 \mathrm{Rn}+0,30$ & 0 \\
\cline { 2 - 4 } & $\mathrm{V}>1,5$ & $-0,050 \mathrm{Rn}+0,30$ & $0,0027 \mathrm{Rn}-0,16$ \\
\hline \hline
\end{tabular}

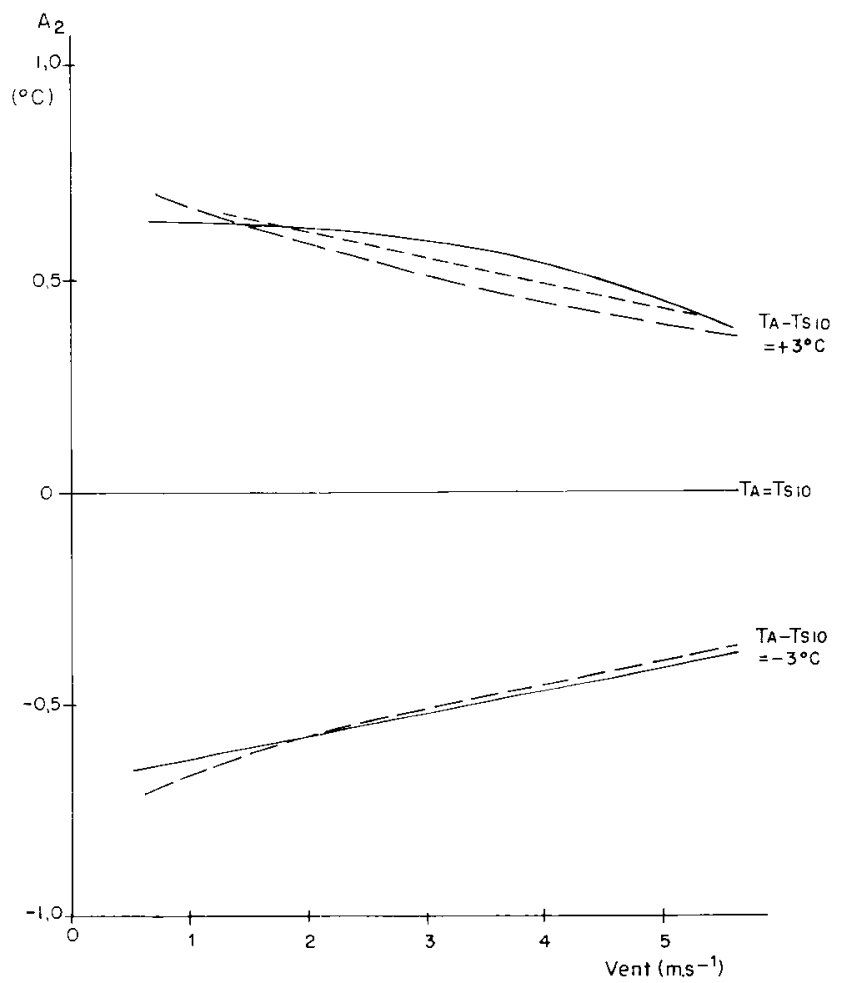

Figure 4

Abaques décrivant la variation du facteur $A 2$ de la relation (4) en fonction des données d'entrée $T_{a}, T_{s / 0}$ et $V$.

(__ $3 \mathrm{~m}$, ..... 1,5 $\mathrm{m}$; - _ surface du sol).

Variation of the $A 2$ factor in relation (4), relative to input data $T_{a}$, $T_{\text {SIO }}$ and $\boldsymbol{V}$.

(__ $3 \mathrm{~m}, \ldots . .-1,5 \mathrm{~m} ;-\ldots$ soil surface).

modèle complet. La régression linéaire de la température minimale de l'air à $1,5 \mathrm{~m}$ prédite par la méthode simplifiée $(Y)$ sur celle qui est calculée par le modèle complet $(\mathrm{X})$, donne :

$$
\mathrm{Y}=0,22+0,97 \mathrm{X} \quad \mathrm{r}^{2}=0,97
$$




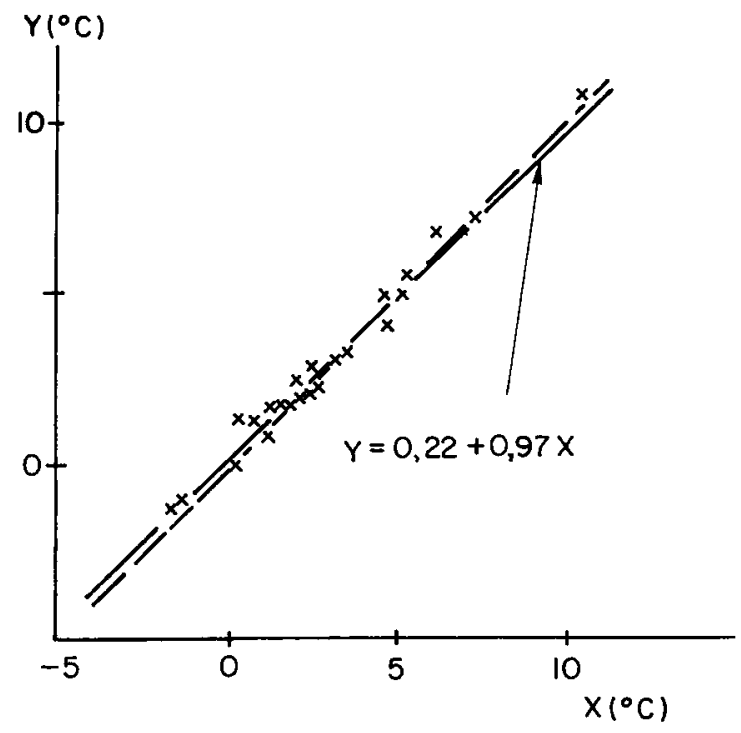

Figure 5

Comparaison entre températures minimales de l'air à $1,5 \mathrm{~m}$ prédites par la méthode simple $(Y)$ et le modèle complet $(X)$, pour 23 nuits de ciel clair à La Minière et Lafrançaise.

Comparison of minimum air temperature at $1.5 \mathrm{~m}$, forecast using the simple method $(Y)$ and the complete model $(X)$ in the case of 23 clear sky nights at La Minière and Lafrançaise.

Les écarts à la $1^{\text {re }}$ bissectrice ne peuvent être attribués à aucun paramètre en particulier.

\section{RÉSULTATS}

Les figures 6 et 7 nous montrent les résultats obtenus à l'aide de cette méthode simplifiée, pour la température de la surface du sol et de l'air à partir des données

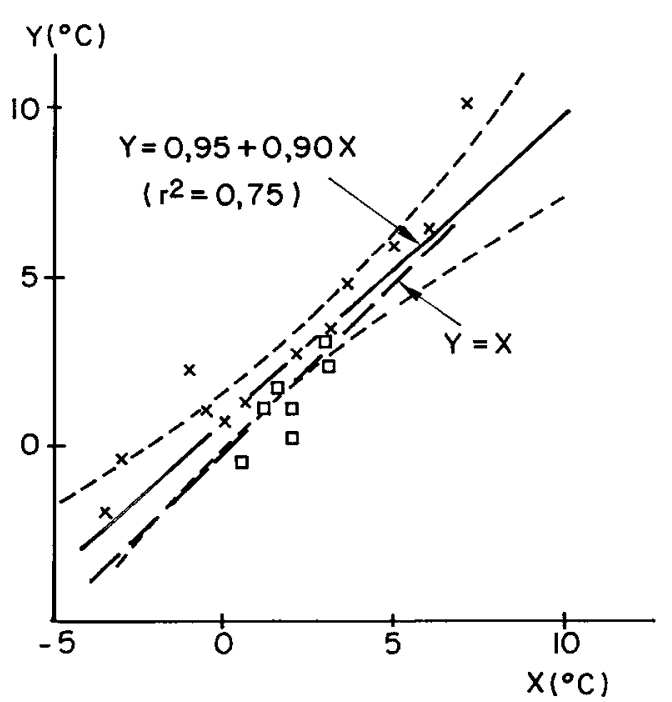

Figure 6

Comparaison entre températures minimales de la surface du sol prédites par la méthode simple $(Y)$ et abservées $(X)$ pour 19 nuits de ciel clair à La Minière ( $\times$ ) et Lafrançaise ( $\square$ ).

intervalle de confiance à 95 p. 100.

Comparison of minimum soil surface temperatures forecast using the simple method $(Y)$, and observed data $(X)$, in the case of 19 clear sky nights at La Minière $(X)$ and Lafrançaise ( $\square$ ). relevées au cours de 2 campagnes de mesures (pour plus de précisions voir CELLIER \& ITIER, 1984) :

- sur le site expérimental de la station de Bioclimatologie de I'I.N.R.A., à la Minière (Yvelines) ;

- dans la plaine du Tarn à Lafrançaise (Tarn-etGaronne).

Dans ces 2 cas, la température de l'air était mesurée à $1,5 \mathrm{~m}$.

Sur les figures $7 a, b, c$, on compare les températures minimales prédites à l'aide de la méthode simplifiée à celles qui sont calculées au moyen d'autres méthodes, que l'on a pu appliquer avec les données dont nous disposions.

- formule de YounG (ElLISON, 1928) : il s'agit d'une formule de type hygrométrique qui donne une prévision de la température minimale sous abri, Tmin :

$\operatorname{Tmin}-\mathrm{Td}=\frac{\mathrm{H}-\mathrm{n}_{1}}{\mathrm{n}_{2}}$

où $\mathrm{H}$, l'humidité relative, et $\mathrm{Td}$ sont mesurées à $18 \mathrm{~h}$ TU et $\mathrm{n}_{1}$ et $\mathrm{n}_{2}$ sont des paramètres à déterminer localement par ajustement sur des données antérieures (ici $n_{1}=60$ et $n_{2}=6$ ).

- méthode de LUTERSTEIN-CHUDNOVSKI (cf. TRIPLET \& ROCHE, 1977, pp. 264-265) qui prévoit la température minimale sous abri, Tmin, à l'aide de la formule suivante :

$$
\mathrm{Tmin}-\mathrm{Ta}=\mathrm{Rn}\left(\mathrm{P} \delta_{1}+\mathrm{R} \delta_{2}\right)
$$

où $\mathrm{P}$ et $\mathrm{R}$ sont des coefficients dépendant de la latitude et de la saison ;

$\delta 1$ et $\delta 2$ sont des facteurs dépendant de l'humidité du sol et de la vitesse du vent à $10 \mathrm{~m}$ du sol au coucher du soleil.

Toutes les prévisions ont été établies sans connaissance préalable des sites, par application stricte des différentes méthodes sur les mêmes cas pour chacune.

Pour tester leur valeur respective, on a recherché les régressions linéaires entre températures observées $(\mathrm{X})$ et températures prédites $(\mathrm{Y})$ par chaque mode de prévision. Elles aboutissent aux relations suivantes :

- méthode simplifiée

$$
\mathrm{Y}=0,26+1,00 \mathrm{X} \quad \mathrm{r}^{2}=0,77
$$

- formule de YOUNG

$$
\mathrm{Y}=0,07+0,82 \mathrm{X} \quad \mathrm{r}^{2}=0,86
$$

- méthode de LUTERSTEIN-CHUDNOSKI

$$
\mathrm{Y}=-0,09+1,05 \mathrm{X} \quad \mathrm{r}^{2}=0,73
$$

Les intervalles de confiance de la moyenne à $95 \mathrm{p}$. 100 sont portés sur les figures $7 \mathrm{a}, \mathrm{b}, \mathrm{c}$.

On voit que les écarts à la mesure sont sensiblement équivalents dans les 3 cas. Toutefois la très bonne corrélation obtenue par la méthode de YOUNG est en partie illusoire, car les coefficients $n_{1}$ et $n_{2}$ de la formule (10) ont été calculés à partir des données expérimentales (Tmin, $\mathrm{Td}$ et $\mathrm{H}$ ) relevées à La Minière et Lafrançaise, dont 75 p. 100 environ sont représentés ici. Il est donc difficile de préjuger de la précision de la méthode appliquée à des cas ultérieurs : on remarquera déjà que la suppression de 25 p. 100 des points (cas où les données disponibles ne permettaient pas d'appliquer simultanément les 3 méthodes) fait passer la pente de la régression de 1,00 à 0,82 . La méthode de LUTERSTEINCHUDNOVSKI est, de son côté, très sujette à erreurs en 

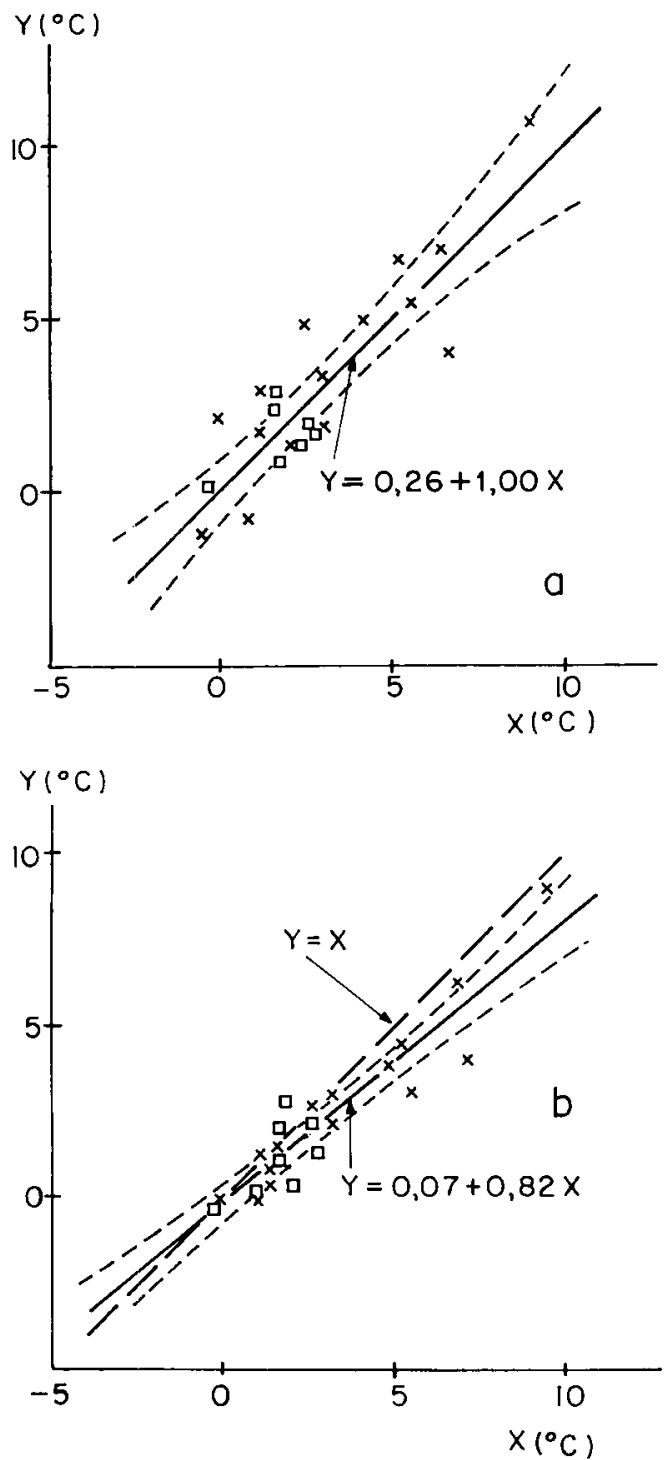

raison de la subjectivité de la répartition des conditions de sol en 5 classes d'humidité (une erreur d'une classe affecte la prévision de $2{ }^{\circ} \mathrm{C}$, environ). Pour ce qui concerne notre méthode simplifiée, par contre, on remarquera qu'elle donne des résultats équivalents à $\mathrm{La}$ Minière et Lafrançaise, sites très différents, sans qu'aucun paramètre ou ajustement local ne soit introduit. Il semble donc que les données d'entrée (§ II.B.) prennent bien en compte l'ensemble des facteurs influençant le refroidissement.

\section{CONCLUSION}

On constate que la qualité de la prévision des températures minimales de l'air obtenue à l'aide de notre méthode simplifiée est au moins équivalente à celle des autres méthodes utilisées classiquement. Il convient de souligner que la qualité est du même ordre pour la température de la surface du sol, qui intéressera plus l'arboriculteur dans la mesure où cette température est plus proche de celle des bourgeons, en conditions de gelée radiative.

Notre méthode présente l'intérêt de ne nécessiter aucun paramètre local ou subjectif - ex : l'humidité $\mathrm{du}$ sol prise en compte implicitement par $\mathrm{T}_{\mathrm{S} 10}-$, dont

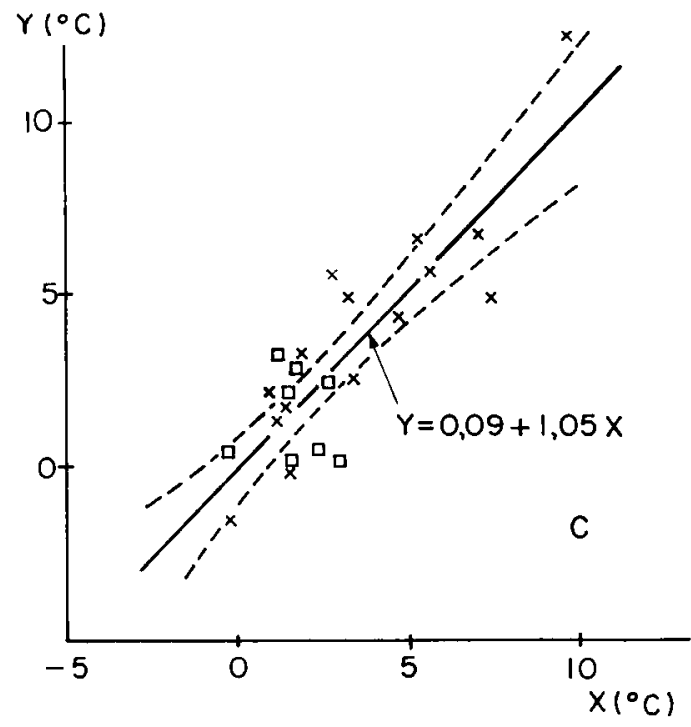

Figure 7

Comparaisons entre températures minimales de l'air à $1,5 \mathrm{~m}$ prédites à l'aide de différentes méthodes $(Y)$ et observées $(X)$ pour 23 nuits de ciel clair à La Minière (X) et Lafrançaise ( $\square$ ).

$a$ : méthode simplifiée

$b:$ formule de Young (N. B. les points portés sur la figure ont servi à ajuster la formule (cf. texte)).

$c$ : méthode de LUTERSTEIN-CHUDNOVSKI.

-- intervalle de confiance à $95 \mathrm{p} .100$

Comparison of minimum air temperatures at $1.5 \mathrm{~m}$, forecast using the different methods $(Y)$, and observed data $(X)$, in the case of 23 clear sky nights at La Minière $(\times)$ and Lafrançaise $(\square)$. a : simplified method

$b$ : YouNG's formula (N.B. Experimental points on the figure were used for the calibration of the formula (cf. text)).

$c$ : LUTERSTEIN-CHUDNOVSKI's method.

l'introduction dans certaines méthodes actuellement utilisées est souvent la source principale des difficultés rencontrées dans l'élaboration d'une prévision. En outre, mise au point à partir d'un modèle numérique qui privilégie les processus d'échange d'énergie au voisinage de la surface du sol - primordiaux en conditions de gelées de printemps -, elle pourrait être utilisée fructueusement en association avec d'autres méthodes - généralement fondées sur des principes très différents - suivant une procédure qui reste à définir.

De plus, elle n'a été testée qu'à partir des résultats expérimentaux en nombre limité par les possibilités climatiques des années concernées. Il convient maintenant de conforter cette méthode en utilisant les importantes séries de relevés météorologiques existantes.

Pour ce qui concerne son application, le problème principal reste certainement la mesure de $\mathrm{Rn}$, qui n'est pas réalisée actuellement sur les postes courants du réseau météorologique, cette mesure restant coûteuse et délicate. Il apparaît pourtant de plus en plus nécessaire de l'introduire au moins au niveau régional, pour de nombreuses applications en agrométéorologie (évapotranspiration notamment).

Le modèle de calcul initial (CELLIER, 1982 : CELLIER \& ITIER, 1984) est susceptible d'améliorations ; on est en droit d'espérer que la méthode simplifiée qui en est 
issue en bénéficiera. Entre autres, il serait intéressant de pouvoir réajuster les prévisions en fonctions d'éventuels changements prévisibles des caractéristiques climatiques (couverture nuageuse, vent, ...).

Reçu le 10 novembre 1983. Accepté le 16 avril 1984.

\section{REMERCIEMENTS}

Je tiens à remercier B. ITIER, tant pour son aide au niveau expérimental que pour les nombreuses discussions que nous avons eues, ainsi que les équipes de l'E.E.R.M. (Etablissement d'Etudes et de Recherches Météorologiques, F 78470 Magny-les-Hameaux) et du S.M.M. (Service Météorologique Métropolitain, F 33700 Mérignac) qui ont mis leurs moyens techniques à notre disposition lors de la campagne de mesures de Lafrançaise.

\section{RÉFÉRENCES BIBLIOGRAPHIQUES}

Barthram J. A., 1964. A method of forecasting a radiation night cooling curve. Meteorol. Mag., 93, 1105, 246-251.

Brunt D., 1941. Physical and dynamical meteorology. Cambridge University Press, New-York, 428 p.

Brutsaert W. H., 1975. On a derivable formula for long-wave radiation from clear skies. Water Resour. Res., 11, 742-744.

Cellier P., 1982. Contribution à la prévision des températures minimales nocturnes en conditions de gelées de printemps. Etude de l'évolution des températures de l'air et du sol au cours de la nuit. Thèse Doct. Ing., INAP-G, Paris, 138 p.

Cellier P., Itier B., 1984. Un modèle de prévision de la température minimale nocturne sous abri et à la surface du sol en conditions de gelées radiatives. J. Rech. Atmos., 18 (1).

Durand R., 1978. Comment déterminer une situation gélive. INVUFLEC, C. R. Journées Nationales, fév. 78, Angers, 29-49.

Ellison E. S., 1928. A critique on the construction and use of minimum temperature formulas. Monthly Weather Rev., 56, 485-495.

Georg J. C., 1978. Techniques of frost prediction. WMO $n^{\circ} 487$, Technical note ${ }^{\circ} 157,5-45$.
Gerbier N., Pedeau R., 1974. Contribution à l'étude des gelées. Météorologie Nationale, N.I.T., Section VII, pièce $\mathrm{n}^{\circ} 21$.

Groen P., 1947. On radiational cooling of the earth's surface during the night, especially with regard to the prediction of ground frosts. Mededel. Verhandel., série B, 1 (9), 35 p.

Jaeger J. C., 1945. Note on the effect of wind on nocturnal cooling. Quart. J. R., Meteorol. Soc., 71, 388-390.

Saunders W. E., 1952. Some further aspects of night cooling under clear skies. Quart. J. R. meteorol. Soc., 78, 603-612.

Sutherland R. A., 1980. A short-range objective nocturnal temperature forecasting model. J. Appl. Meteorol., 19, 247-255.

Sutton O. G., 1953. Micrometeorology. Ed Mc Graw Hill, London, $333 \mathrm{p}$.

Tabard P., 1978. Etude des moyens de lutte contre les gelées. INVUFLEC, C. R. Journées Nationales, févr. 1978, Angers, 125-150.

Triplet J. P., Roche G., 1977. Météorologie générale. Ed. Ecole nationale de la météorologie, $317 \mathrm{p}$.

De Villele O., 1980. La lutte contre les gelées : choix d'une stratégie adaptée. Vititechnique, 36, 11-15. 